\title{
Arborescences
}

Revue d'études françaises

\section{Discours épilinguistiques en francophonie manitobaine : une vue d'ensemble}

\section{Sandrine Hallion}

\section{Numéro 1, mars 2011}

Identités linguistiques, langues identitaires : à la croisée du prescriptivisme et du patriotisme

URI : https://id.erudit.org/iderudit/1001940ar

DOI : https://doi.org/10.7202/1001940ar

Aller au sommaire du numéro

Éditeur(s)

Département d'études françaises, Université de Toronto

ISSN

1925-5357 (numérique)

Découvrir la revue

Citer cet article

Hallion, S. (2011). Discours épilinguistiques en francophonie manitobaine : une vue d'ensemble. Arborescences, (1), 0-0. https://doi.org/10.7202/1001940ar

\section{Résumé de l'article}

Tout locuteur formule des jugements de valeur sur la ou les langues qu'il parle ou qui l'entourent. L'examen de discours épilinguistiques dans le contexte de la francophonie minoritaire du Manitoba permet de répertorier un certain nombre de réflexions qui forment un réseau de représentations sur une gamme de pratiques linguistiques allant de l'emploi du « français correct » à celui de l'anglais, en passant par l'usage de toutes les variétés linguistiques de la " zone vernaculaire " qui s'étend entre ces deux pôles. L'analyse de ces discours donne une vue d'ensemble des sentiments des francophones vis-à-vis des langues ou des variétés de langues qu'ils emploient ou qu'ils côtoient. Il s'agira dans cet article de présenter ce panorama à partir de l'étude de trois types de corpus : 1) des entrevues semi-dirigées ; 2) des témoignages de jeunes francophones du secondaire recueillis sur un forum de discussion sur Internet ; 3) des documents écrits du type introductions de glossaire, articles scientifiques et articles de journaux. Cet examen mettra au jour des perceptions courantes, prévisibles et récurrentes, observées ailleurs en francophonie. Le rôle que peuvent jouer ces représentations de la langue dans le processus de changement linguistique sera finalement évoqué.
Tous droits réservés @ Département d'études françaises, Université de Toronto, 2011
Cecument est protégé par la loi sur le droit d'auteur. L'utilisation des services d’Érudit (y compris la reproduction) est assujettie à sa politique d'utilisation que vous pouvez consulter en ligne.

https://apropos.erudit.org/fr/usagers/politique-dutilisation/ 


\title{
DISCOURS ÉPILINGUISTIQUES EN FRANCOPHONIE MANITOBAINE \\ Une vue d'ensemble
}

\author{
Sandrine Hallion \\ Collège universitaire de Saint-Boniface
}

\begin{abstract}
«Le stéréotype est de longue durée, imperméable à l'expérience, même contraire à son contenu, gardant sa force persuasive même à travers des générations. »
\end{abstract}

(Bochmann, $2001:$ 101, note 1)

\section{Introduction}

Les représentations épilinguistiques, cet « ensemble ondoyant des conceptions ordinaires que se font les locuteurs des langues qu'ils parlent », pour reprendre la définition qu'en donne Paul Laurendeau (2004: 431), se traduisent au Manitoba, comme ailleurs, par différents types de discours dont l'analyse permet de documenter les manifestations des jugements esthétiques sur la langue française en contexte francophone manitobain. L'examen de ces discours permet également de faire l'inventaire de la terminologie employée au Manitoba francophone pour tenter de classer et de caractériser diverses variétés de français dont on peut chercher en outre à cerner les particularités. Comme le rappelle Lafontaine (1997 : 59), l'analyse « classique » des attitudes linguistiques - c'est-àdire celle qui s'appuie sur les sentiments des locuteurs vis-à-vis des variétés linguistiques qu'ils emploient et/ou avec lesquelles ils sont en contact plus ou moins étroit - a ses limites dans la mesure où elle ne donne qu'un aperçu « grossier » de la situation à l'étude et que les opinions qu'elle révèle sont destinées à se dissoudre dès lors qu'elles sont soumises à une analyse plus fine. Cette méthode reste toutefois valide pour « débroussailler un nouveau terrain » (Lafontaine 1997 : 59), afin de mettre au jour les stéréotypes présents dans une communauté linguistique donnée. C'est dans cette perspective de défrichement que se situe cette étude.

Le français au Manitoba se caractérise par ce qu'on a coutume d'appeler sa position «minoritaire», adjectif renvoyant à une réalité statistique puisqu'un peu moins de $4 \%$ de la population totale de la province déclarait le français comme langue maternelle unique au 
recensement de 2006. Cette position confère en contrepartie à la langue anglaise le statut de langue majoritaire. Ce concept de «minoritaire » renvoie également à une réalité sociale, le français restant somme toute, à l'heure actuelle, et malgré la percée de cette langue dans la sphère publique, un code bien moins légitime que l'anglais à l'échelle provinciale. Cet environnement bilingue a naturellement des conséquences sur les variétés de français parlées dans la province qui témoignent à divers degrés de phénomènes d'emprunts et d'alternances de langues. Les variétés mixtes qui découlent du contact linguistique sont, à quelques exceptions près, perçues négativement, ce qui n'a rien de surprenant en situation de diglossie. D’autre part, la francophonie manitobaine bénéficie de bonnes assises institutionnelles, médiatiques et scolaires qui forment un réseau de diffusion de représentations normatives endogènes - lorsque le «bon usage » émane de représentants actifs ${ }^{1}$ appartenant à la communauté linguistique (des locuteurs Franco-Manitobains) - et exogènes - lorsque le «bon usage » émane de représentants actifs extérieurs à la communauté linguistique (essentiellement des locuteurs venus du Québec, de l'Europe et de l'Afrique francophones). L'existence d'un pourcentage grandissant d'immigrants francophones venus d'Europe, et surtout d'Afrique, impose également la présence, au sein même de la communauté linguistique, de modèles passifs externes souvent valorisés. Dans ce contexte, les discours épilinguistiques se focalisent sur certains éléments courants, prévisibles $^{2}$ et récurrents qui tissent un réseau de représentations sur la langue.

\section{Les corpus exploités}

Les corpus exploités pour cette étude sont de trois types et se composent:1) de transcriptions de corpus oraux constitués à partir d'entrevues semi-dirigées comportant des questions classiques susceptibles de provoquer des discours épilinguistiques ; 2) de témoignages de jeunes francophones $\mathrm{du}$ secondaire recueillis sur un forum de discussion accessible sur le site Internet de Facebook; et 3) de documents écrits du type introductions de glossaires, d'articles scientifiques et d'articles de journaux.

Les corpus oraux ont été recueillis auprès d'un échantillon de 33 locuteurs francomanitobains entre 1995 et 1997. Notons que l'échantillon de locuteurs interviewés comprend un

\footnotetext{
${ }^{1}$ Les représentants actifs sont les membres de l'élite culturelle, les journalistes, les enseignants et professeurs, agents légitimes de diffusion de la norme. Les représentants passifs sont de simples locuteurs de la communauté dont les usages sont érigés en modèles.

2 L'adjectif courant renvoie à la fréquence de ces représentations au sein de la communauté linguistique, l'adjectif prévisible au fait qu'elles ont été observées ailleurs en francophonie.
} 
nombre élevé de personnes ayant suivi une formation postsecondaire. Le questionnaire utilisé lors des enquêtes contient un certain nombre de questions propres à créer des conditions d'insécurité linguistique - d'autant que l'intervieweuse est Française - et à susciter la verbalisation de stéréotypes linguistiques courants au sein de la communauté francophone locale. Les questions posées aux participants sont, par exemple : Estimez-vous avoir une bonne compétence en français? Comment qualifieriezvous le français que vous utilisez? Quelle est votre définition du "bon français », du "français correct »? Êtes-vous conscient des écarts qui existent entre le français du Manitoba, celui du Québec, celui de France? Vous sentez-vous menacé par l'anglais?

À mi-chemin entre l'oral et l'écrit's les témoignages de jeunes Franco-Manitobains accessibles sur un forum de discussion portant sur des questions reliées aux langues et à l'identité constituent la deuxième source de données exploitées. À la fin du mois de mai 2008, une élève du Collège LouisRiel, école secondaire francophone de Winnipeg, cherche à relever le pari lancé par un ami : trouver en un mois 50 élèves de son école (sur environ 650-700 élèves) qui déclarent se sentir plus à l'aise en français qu'en anglais et les inviter à se manifester sur le forum ouvert à cet effet sur Internet. Les interventions des élèves témoignent notamment de la perception négative dont font l'objet les variétés mixtes de français issues du contact intense avec l'anglais. Ce genre de discours constitue un matériau intéressant dans la mesure où il provient de la génération francophone montante, élite de demain, et qu'il permet de juger de la pérennité des représentations épilinguistiques. Il révèle également que, comme le constate Annette Boudreau pour l'Acadie (1991), ces jeunes ont une forte conscience linguistique qui est due au contexte de réflexion métalinguistique constante en milieu francophone minoritaire.

Enfin, les documents écrits se composent d'introductions de glossaires ou de recueils de mots ou d'expressions. Ce sont également des articles scientifiques ou de journaux abordant des questions linguistiques de manière plus ou moins objective. Les corpus écrits ont pour principal intérêt de constituer des vecteurs durables - «les paroles s'envolent, les écrits restent » - et légitimes - ils émanent des élites culturelle et universitaire locales - de représentations et de reproduction des stéréotypes linguistiques.

\footnotetext{
${ }^{3}$ La remarque suivante illustre bien le caractère hybride de ce médium : « [...] sur le web, j’écris comme je parle » (http://www.new.facebook.com/wall.php?id=18686351606)
} 


\section{Perceptions linguistiques au Manitoba}

Il est possible de présenter brièvement et schématiquement la situation du français au Manitoba en signalant l'existence d'un éventail de pratiques linguistiques allant de l'emploi du « français correct », la variété prestigieuse et légitime, à celui de l'anglais, code linguistique majoritaire assujettissant et «abâtardissant » le français. Entre ces deux pôles, se situe ce que j’appellerai la «zone vernaculaire », soumise à la double pression de ces pôles. Les regards portés sur cette gamme d'usages par des locuteurs francophones de la province permettent de révéler un certain nombre de représentations courantes dans la francophonie manitobaine.

Deux phénomènes récurrents ont été mis au jour par les recherches menées sur les attitudes linguistiques en contexte multilingue. Notons que cette notion de multilinguisme renvoie à la présence de codes linguistiques différents mais également à celle de variétés d'une même langue en concurrence. D’une part, on observe chez les locuteurs de la variété dominée une autodépréciation de leur vernaculaire, dévalorisation interne qui est symptomatique d'une situation d'insécurité linguistique. D’autre part, cette même variété dominée et non légitime fait l'objet d'une perception positive, souvent de type affectif, et s'associe à des valeurs comme la sympathie, la douceur, etc. Elle est aussi jugée comme la variété appropriée dans certains contextes, surtout informels. Les locuteurs lui confèrent donc un «prestige latent ». Dans le même temps, d'autres valeurs, comme l'élégance, la clarté, la pureté, etc., sont ordinairement associées à la variété prestigieuse légitime (cf. Lafontaine $1997: 58)$.

Ces deux tendances se retrouvent au Manitoba. Les trois extraits suivants témoignent du phénomène d'autodépréciation du vernaculaire.

(1) Moi, je parle dans un registre... un registre de langue euh... populaire, familier... [...] au meilleur de mes capacités, c'est populaire et familier [...]. Je peux pas parler comme ceux qui passent à la Radio-Canada ; parce que je suis pas assez instruit et puis, les influences de l'anglais est énorme! (I314)

(2) I. - Comment tu le qualifierais, ton français ?

P. - Oh! [Rire nerveux]. Je pense que, «médiocre », je peux même dire. (I30)

(3) ...les standards sont encore une fois si bas, on se contente d'un français médiocre, entrecoupé par des expressions et même des phrases en anglais, ce qui prouve le manque d'aisance en français... (Forum, 8 juin 2008)

La deuxième tendance se trouve illustrée par la série d'extraits reproduite ci-dessous. Dans l'extrait (4), le participant associe sa pratique du vernaculaire à une pratique positive (fierté) qu'il relie

\footnotetext{
${ }^{4}$ Informateur numéro 31 (I31). Tous les exemples accompagnés de ce type de référence sont issus de Hallion (2000).
} 
à l'estime qu'il ressent pour sa famille. L'analogie établie dans l'extrait (5) montre que la variété vernaculaire suscite des associations valorisantes de nature affective. Le vernaculaire est aussi une variété de français adéquate dans les contextes informels (6) alors que la variété prestigieuse est associée à des valeurs comme la clarté (7) ou la beauté (8).

(4) Mais... je suis très fier du français que je parle... parce que, je suis fier de ma famille, et puis... ceux qui... je ne dis jamais que je ne parle pas le bon français (I31)

(5) [notre langage] c'est un langage doux, qui coule comme la rivière et le vent de l'Ouest là (I5)

(6) le bon français c'est ce que tu parles dans ton milieu. [...] mon français est supérieur, quand je suis dans ma famille; mon français est bien quand je parle avec mes amis (I31)

(7) Disons, toi tu es Française, tu viens de la France, pour moi tu parles très bien. Tu t'exprimes très bien. (I5)

(8) je trouve beau ! le français de la France. C'est celui-là que j'aime ! (I18).

Comme je l'ai déjà signalé, les discours épilinguistiques se focalisent en outre sur certains éléments courants, prévisibles et récurrents dont on peut proposer le classement suivant : (i) la dévalorisation des variétés prestigieuses; (ii) la recherche de l'intercompréhension, du mot juste et de l'équilibre ; (iii) la dévalorisation des variétés anglicisées et la valorisation des archaïsmes ; (iv) la revendication des variétés anglicisées. Chacune de ces catégories sera détaillée dans les sections qui vont suivre.

\section{- Dévalorisation des variétés prestigieuses}

Le pluriel est ici employé dans la mesure où il n'y a pas de consensus dans l'identification d'une variété modèle valorisée. Il s'agit tantôt d'une variété exogène venue de France (9) ou d'autres pays francophones (10 et 11), tantôt d'une variété globalisante identifiée sous l'étiquette « français international » (12), tantôt de variétés canadiennes endogènes comme « le bon français du Québec » (13) ou de la variété emblématique appelée « le français de Radio-Canada » (14).

(9) « le bon français »... mais on pense toujours aux Français, vous avez un excellent français! (I33)

(10) mon mari a une belle prononciation. Lui... lui... euh... sa mère... bien, sa grandmère et son grand-père sont venus de la Suisse, alors oh! c'est beau ! [Rires]. Et puis mes enfants ont pris ça de... de... de lui ! (I18)

(11) La norme c'est... on devrait être... les Africains, eux et les pays fran... français en Afrique, eux $[\ldots]$ eux ils parlent un très bon français aussi, beaucoup moins d'erreurs que... mais ils ont pas le... autant d'influence que nous! (I31)

(12) I. - Quelle est pour vous, la définition du «bon français»? P. - Bien... en ce moment, c'est... c'est le français international. Qu'on... qui est 
reconnu comme étant, le «bon français ». [...] Alors, le «bon français » semble être celui qui... qui est international aussi, alors tout le monde peut... comprendre, oui. (I18)

(13) Le Québec a... a dit: «Le bon français, c'est le français de France », et nous disons ben, le... c'est le bon franç... c'est du Québec, nous autres a d... c'est un peu, le bon français de France, on avait moins de contacts avec la France que le Québec; alors c'est un peu ça là, tu sais... [...] le bon français du Québec. (I10)

(14) Juste pour répondre, je dirais peut-être, quelqu'un qui comm... le français de RadioCanada, ça c'est le français qui serait peut-être... Parce qu'il y a... il y a pas d'erreurs, c'est un français peut-être moins... plus neutre si tu veux, il y a pas le... l'intonation régionale... Puis ça serait peut-être... tu sais, si on voulait apprendre le français, ça serait peut-être le meilleur... tu sais. (I16)

La valorisation des variétés prestigieuses, en particulier lorsqu'elles ont une origine exogène, connaît toutefois des limites. Ces variétés peuvent être condamnées lorsque leur valorisation s'accompagne d'une dépréciation du vernaculaire venue de l'extérieur. Le locuteur perçoit cette dernière comme une atteinte au lien affectif et identitaire qu'il entretient avec la variété dont il fait usage. Plusieurs facteurs contribuent à la perception négative des variétés prestigieuses. Certains dénoncent le laxisme français en matière d'emprunts à l'anglais. D'autres invoquent l'inauthenticité et la préciosité de variétés prestigieuses, mais inadéquates en contexte local. Ces différentes perceptions seront maintenant tour à tour considérées.

\section{- Lien affectif et identitaire avec le vernaculaire}

Certains discours de revendication illustrent la pression normative qui s'exerce sur des variétés locales dévalorisées et la recherche d’une légitimité, tout aussi valable que celle accordée aux variétés prestigieuses, au nom de l'attachement affectif et identitaire (15 et 16).

(15) j'aime pas qu'on me dévalorise ${ }^{5}$ pour autant. Tu sais, je... je veux rester fière de... de ce... de ce que je suis là! Pis je suis certaine que les Français que... qui viennent ici, pis qu'on leur dit : «Oh! vous parlez un français qui est pas... compréhensible, trop trop pour ici », c'est pareil pour eux. Tu sais, ils vont dire : «Ben - tu sais - mais moi c'est du vrai français ». Mais, ton vrai français... pour moi là, mon vrai français c'est le mien. (I5)

(16) j'aime le français de mon père là, parce que c'est à lui ! [Petit rire]... puis... tu sais, c'est... Pourquoi juger ou rab... rabaisser... son français ? (118)

\footnotetext{
${ }^{5}$ Les caractères gras sont utilisés pour mettre en relief la récurrence de certains termes et de certaines opinions.
} 
(17) quand il y a du monde qui dit : «Oh ben, c'est... c'est pas comme ça, c'est... on dit 'la' telle chose ». Peut-être ils pensent qu'ils aident mais $1 . .$. normalement, le façon qu'ils disent, moi je me trouve c'est... une façon de... d'abaisser quelqu'un, pis moi, forget it là : «Si tu aimes pas mon français, je vais vous parler en anglais ». (I19)

L'extrait (17) montre en outre que le poids de la norme peut contribuer à faire «basculer» le locuteur francophone dans le pôle opposé du continuum linguistique, c'est-à-dire l'anglais. Ce type de conséquence se retrouve ailleurs en francophonie minoritaire au Canada comme en témoignent les remarques suivantes formulées pour le Nouveau-Brunswick: «Si [la] variété de langue [des francophones qui parlent un français plus ou moins éloigné du standard] est dévalorisée, ils pourraient se tourner vers l'anglais, où ils savent que leurs productions ne seront pas jugées et qu'ils peuvent s'abriter derrière le fait que ce n'est pas leur langue maternelle » (Boudreau et Gadet 1998).

\section{- Perception d'un laxisme français en matière d'emprunts à l'anglais}

Le discours qui dénonce l'anglomanie française (18) est récurrent. Il s'accompagne d'exemples typiques, «rituels» (Laurendeau 2007 : 33), pris dans le lexique. Les plus fréquemment cités pour illustrer « les niaiseries qui se passent en France» (I31) sont les emprunts à l'anglais weekend et parking.

(18) par rapport à vos anglicismes, flagrants là, je pense que [notre français] est supérieur, mais... parce que jamais moi, je dirais : «Tu vois le parking là... », et pis «le jogging », et pis «le... building», pis «le smoking», pis «le...» jamais jamais qu'on parlerait comme ça nous, c'est pas du français pour nous ça. Alors... de ce point de vue-là, on est peut-être plus euh... plus pur (I20)

Les francophones jugent souvent que, de ce côté-là, le Québec fournit un modèle plus valable que celui provenant de la France :

(19) j'écoute là... la radio euh... la télévision française puis, tout ce bagage de vocabulaire an... anglais, américain! Je dis, ils se forcent vraiment pas pour... le Québec se force un peu plus que la France pour inventer des mots fran... francophones, puis je les félicite pour ça puis je voudrais... que... qu'on le dise aux Français, que c'est dommage qu'ils ai... ils n'aident pas... ils ne nous aident pas en faisant ça (I10)

\section{- Conscience que le « bon français » est une abstraction de la langue écrite}

Pour certains, le «bon français », le «français correct» relève du fantasme puisqu'il ne se réalise complètement chez aucun des locuteurs de cette langue (20). Dans l'extrait (21), cette abstraction, que le français de l'Académie représente par excellence, est perçue comme un code non fonctionnel à l'oral :

(20) le français correct n'existe nulle part, finalement... (I20) 
(21) pour moi, il y a pas de bon français [...]. Je suppose que, je pourrais dire, bon ben : "Le français de l'Académie française, ça c'est... tu sais - ça c'est le... », comme ça. Mais, qui parle comme ça, tu sais? (I16)

\section{- Le sentiment d'une préciosité, d'une inadéquation au contexte local des variétés prestigieuses}

La variété prestigieuse, qui dans l'extrait (22) est identifiée à «l'usage parisien », apparait également inadéquate en contexte local. Ce modèle s'accompagne d'une préciosité que l'on rejette et que l’on déprécie :

(22) [...] le français canadien n'a peut-être pas le panache du caniche qu'est le Parisian French, mais il est certain qu'on ne prend pas au Canada l'usage parisien comme la norme (Léveillé, 2005 : 84).

Dans un même ordre d'idées, l'extrait (23) dénonce l'atténuation excessive de traits linguistiques (ici phonétiques) caractéristiques de la variété locale de français dans le discours de certains représentants de l'élite locale. Cet évitement, perçu comme une sorte de trahison, semble inadapté, même en situation formelle :

(23) On n'a qu'à allumer le téléviseur sur notre unique chaine française locale pour entendre tous les soirs de la semaine à notre bulletin de nouvelles locales, l'animatrice, originaire d'ici, prononcer le nom de notre province à la française, ou peut-être à la québécoise. Bon, je comprends qu'un bulletin de nouvelles ne se fasse pas lire en « joual» de La Broquerie ou de Sainte-Anne, mais quand même le «a » à la fin de Manitoba n'est pas celui de patate ou de tache (Le Réveil, 2005 : 20).

L'emploi de la dénomination joual pour désigner des variétés topolectales, et vraisemblablement sociostylistiques, du français du Manitoba peut ici surprendre. Traditionnellement associé au parler populaire de Montréal, ce terme à connotation péjorative connait pourtant un sens étendu signalé notamment par Claude Poirier dans son Dictionnaire historique du français québécois. Il y renvoie en effet à « toute variété linguistique considérée comme déviante par rapport à une norme donnée » (1998: jouah. C’est dans ce sens qu'il est employé dans le corpus examiné.

\section{- Recherche de l'intercompréhension, du mot juste et de l'équilibre}

Un deuxième élément qui revient régulièrement dans les discours est celui qui présente la recherche du mot juste et de l'équilibre comme les garants de la qualité de la langue. On ne condamne pas ouvertement l'habitude française de parler trop vite qui, dans les extraits (24) et (25), est davantage analysée comme une faiblesse de l'auditeur que comme une incompétence du présentateur (il ne faut pas perdre de vue que l'intervieweuse est d'origine française); mais on 
valorise l'intercompréhension (26) et le parler des locuteurs qui réussissent à trouver un savant dosage dans le choix des mots et des expressions (27).

(24) Moi j'ai parfois de la difficulté... à comprendre les présentateurs ou les annonceurs. D'abord, leur débit est tellement rapide... l'accent est différent, qui faut... [il fait] qu'on est obligé de... d'écouter avec plus de... d'attention. Et, des fois les mots s'agrippent l'un après l'autre, pis on manque... on manque le sens d'une phrase... (I7)

(25) quand on... on regarde les programmes de télévision qui viennent de France là, on a de la difficulté à comprendre, parfois. Je sais pas si ils parlent trop vite, ou bien donc... que i... articulent pas assez, mais on a de la misère pis ils ont des... des accents qu'on... qu'on est pas habitué. (I9)

(26) Pour moi, la définition du français correct, c'est d'être communiqué puis s'entendre d'une personne à l'autre. Si on comprend qu'est-ce que l'autre personne dit, pour moi c'est ça qui est très important. (I13)

(27) [le «bon français»] c'est pas... pas nécessairement parler à la française, mais un français avec les... les mots justes, et puis euh... on dit que... tu sais, il y a des expressions pour... pour tout exprimer, alors, c'est quelqu'un qui a un beau... [un] bon vocabulaire... qui peut varier ses expressions puis... et puis, pas... pas bourré d'anglicismes. (I9)

\section{- Dévalorisation des variétés anglicisées et valorisation des archaïsmes}

La remarque du dernier extrait permet d'enchaîner sur la troisième tendance que l'on peut identifier dans les discours et les écrits consultés. Il s'agit de celle qui consiste à condamner les variétés anglicisées du français local, tendance qui va bien souvent de pair avec la valorisation des survivances d'un état plus ancien de la langue française, liée au fantasme de la pureté des origines ${ }^{6}$. Cette association entre anglicismes à proscrire et archaïsmes à préserver est ancienne dans l'histoire du prescriptivisme au Canada français7. Au Manitoba, on la retrouve dans des introductions de glossaires ou de recueils de mots. Liliane Rodriguez remarque dans son recueil d'archaïsmes courants au Manitoba français qu'il faut «chérir les premiers [les archaïsmes] pour mieux guérir les seconds [les anglicismes]»(1984: 88-89). En 2006, dans l'introduction à son dictionnaire d'anglicismes, Antoine Gaborieau reprend ce leitmotiv en préconisant la réhabilitation des archaïsmes, ces vestiges

\footnotetext{
${ }^{6}$ Le mythe de la pureté conservée par le français canadien apparaît dans une entrevue récente (octobre 2008) avec JeanDenis Gendron. On y trouve, dans le sous-titre, la remarque suivante : «Les Québécois ne parlent pas «mal». Leur accent est aussi pur que celui qui prévalait à la cour de Louis XIV. En fait, ce sont les Français qui ont changé d'accent [...] 》 (Entrevue avec Jean-Denis Gendron : 26). Un locuteur franco-manitobain reprend cette opinion commune : «Il y a des gens qui disent que le Qué... le... le français au Québec... des gens qui disent... c'est une opinion, je pense... que le français du Québec c'est le français qui est peut-être le plus pur parce que, il a pas beaucoup changé depuis des centaines d'années. Tandis que... en France, il a... il a évolué ; tandis qu'au Québec, le vieux fran... c'est beaucoup comme du vieux français qui se parlait autre... euh autrement... autrefois au Québec... euh en France, excuse (Hallion, 2000 : I25).

${ }^{7}$ Entre autres, Ernest Gagnon signalait en 1892: «Les archaïsmes, que nous devons conserver comme de vieux joyaux de famille, et les anglicismes, dont nous devons nous débarasser [sic] avec le plus d'application possible » (cité dans Fournier, 2001-2002).
} 
du « vieux français » qui « [font] partie de notre héritage culturel français et québécois » (2006:11) et déplore «les anglicismes qui pullulent dans notre parler, à tel point que nous n'employons presque aucune phrase qui ne soit farcie de ces expressions étrangères à notre patrimoine culturel » (2006 : 13).

En situation d'entrevue, le sentiment vis-à-vis des archaïsmes est parfois esquissé comme le montre l'extrait (28); cette composante de la variété locale semble lui conférer une plus grande légitimité surtout du fait qu'elle permet de rattacher cette variété à son origine française et à atténuer le facteur « contact linguistique » comme principal facteur de différenciation linguistique.

(28) mon père... parle un « canayen » là, fort! Et puis... hum... je sais pas si les Français de la France comprendraient ce qu'il dit... Mais, pourquoi est-ce qu'il parle comme ça ? C'est... je suis pas certaine mais, j'ai... une fois, j'ai ouvert le livre de Tristan et Is... Tristan et Iseult, mais il y avait du v... vieux français comme ça de... que mon père dit! Je me demandais : «Est-ce que ça vient de la France ? Est-ce que c'est ça ? Est-ce que c'est du vieux français ? ». [...] il y a des gens qui vont dire : «Oh! mais, c'est pas du bon français ça !». Moi, je dis : «Hum!». C'est... c'est pas bien! C'est... il me semble c'est ju... c'est... c'est leur français ! [...] Alors, peut-être qu'ils ont un... un français qui est... qui est plus vieux... parce que, ils sont pas tellement assimilés mes parents! Ma mère a de la difficulté à parler en anglais! (I18)

Dans le contexte manitobain, les phénomènes de mélanges linguistiques liés au contact intense du français avec l'anglais concernent tout un chacun, à divers degrés. La condamnation des variétés anglicisées du français est assez générale dans les discours (29). Ces variétés sont souvent identifiées sous l'appellation de « franglais » (30).

(29) je pense que quand tu parles le français, tu devrais être capable de le parler sans constamment te lancer des expressions anglaises. Ça c'est pas un bon français! (I32)

(30) notre fran... franglais... à part d'avoir des mots anglais, on a toute des tournures de phrases anglaises puis les... les personnes s'en rendent pas compte! On leur dit: «Mais c'est pas français ça!» (I10)

L'usage des variétés anglicisées les plus stigmatisées s'associe à des facteurs géographiques et sociaux : ce sont des variétés rurales (31) dont l'emploi est plus fréquent chez les personnes moins éduquées (32).

(31) Et il y a des villages où le français est... est très... très pauvre. Parce qu'il est... il est très très assimilé, il y a beaucoup beaucoup d'anglais, et il y a une mauvaise prononciation, c'est-à-dire qu'il y a une prononciation qui est difformée, qui... qui n'est même pas du vieux français, qui est une prononciation... je sais pas, anglaise... Alors ça c'est pas... bon (I11)

(32) dépendant de l'éducation des parents... le français des enfants varie. Et puis aussi de ce qu'ils peuvent assimiler à l'école... Si ils font... juste un peu à l'école, puis un peu à la maison, puis partout ailleurs c'est anglais, leur français est encore moins... moins bon. (I14) 
L'emploi d'anglicismes ou du «franglais» s'associe en outre à une faiblesse (33), à une paresse intellectuelle (34) ou à l'indifférence (35) :

(33) Je veux dire faut que je fasse attention à mes anglicismes. Ça c'est un... ça c'est une faiblesse, puis je me dis... j'ai pas d'excuse. Mais, des fois, le mot français ne vient pas. (I6)

(34) la qualité du... du français laisse beaucoup à désirer et puis c'est... c'est... du franglais qu'on dit! Oh c'est terrible! Moi, je lutte contre ça, je trouve que c'est... c'est massacrant. Euh c'est... quand on entend des mots anglais, les mots... euh c'est une paresse puis aussi, une paresse intellectuelle à se forcer, trouver les bons termes alors on va au plus... au mot qui vient le plus vite, c'est le mot anglais (I10)

(35) je vais faire certaine que cette phrase fait du sens : Si j'aurais payé attention à c'qu'on parlait de, j'aurais tourné rouges.

C'est le langage moyen des franco-manitobains [sic] qui sont indifférents face à leur français ! [...] UN ANGLICISME, CE N'EST PAS DU FRANÇAIS!! Si vous ne faites pas d'effort de parler français, en français, c'est-à-dire sans le parsemer d'expressions anglophones ou d'anglicismes, ne vous dites pas plus à l'aise en français qu'en anglais. (Forum, 21 juin 2008)

\section{- Revendication es variétés anglicisées}

Si certains luttent contre la mixité linguistique (cf. le témoignage en (34)), d'autres constatent que les mélanges de codes font naturellement partie de la réalité linguistique qu’ils vivent (36 et 37), sans pour autant chercher à passer à l'étape de revendication de ces usages.

(36) Ici, au Manitoba, si on peut pas s... trouver le mot, on le dit en anglais! C'est... tout simplement. (I23)

(37) Mais c'est parce que c'est tellement... c'est devenu tellement naturel! Tu viens que tu as... tu as... tu as pas... plus ou moins une maittrise de la langue française, pis plus ou moins une maitrise de la langue anglaise... pis quand tu parles, ben! tu sautes d'un à l'autre (I32)

Dans l'extrait suivant (38), l'anecdote racontée révèle l'intégration profonde de certaines expressions en anglais dont on ne connaît pas l'équivalent en français.

(38) chez nous on avait des anglicismes, d'abord hein. Alors, je les ai pas tous perdus. Je suis arrivée à la supérieure générale, une fois, jeune novice moi pis, mon père il voulait que j'appelle plus souvent chez nous. Puis, il m'a dit, il dit : «Reverse the call... Il dit pis, on va le payer nous autres ». Fait que j'arrive à la... la... à la supérieure générale, moi pis... me rappelle était couchée [...]. Pis je dis : «Papa aimerait bien ça que j'a... que j'appelle plus souvent à la maison, pis reverse the call ». Oh ! [ma chère] ! Oh ! elle, c'était une Québécoise! [Rires]. Je me suis lé fait dire! Puis «reverse the call», moi j'avais jamais rien entendu d'autre! Je savais même pas comment dire ça en français, hein. (I6)

Le discours de revendication de l'usage de variétés de français anglicisées est très rare en situation d'entrevue où prime le sentiment que ces variétés n'ont aucune légitimité. Lorsqu'il se

${ }^{8}$ On a affaire ici à ce que Paul Laurendeau appelle un « exemple rituel» (2007 : 33). 
rencontre, il s'appuie sur l'argument de l'importance de la conservation de l'usage du français, quel que soit son niveau de correction («Peut-être je le re... je parle le français comme une vache, mais au moins je le parle », I19), et il met de nouveau en relief le fait qu'une trop grande recherche de la perfection peut mener au basculement vers l'usage exclusif de l'anglais (39).

(39) en jeunesse, qu'est-ce qui m'achalait le plus - pis j'ai vu ça un petit peu [de] même ici, quand je travaille au Musée - si je parlais, pis il y a quelqu'un qui me corrigeait! «Okay, fine, je vais parler en anglais». Tu sais. Pis c'est ça que... j'haïssais ça en jeunesse, pis je dis: "Écoute», tu sais, «dans certaines cas on devrait être content qu'on peut parler en français. Mal, bien, in between, ça fait [pas de, bout] [différence]... c'est... c'est important, mais c'est... on... on se comprenne ». (I19)

Ce discours de revendication linguistique et identitaire se retrouve parfois chez les jeunes dans certains écrits de nature polémique, comme des articles de journaux (40) ou des œuvres fictives de création littéraire (41).

(40) J'ai dû subir tout au long de ma jeunesse, de mes collègues de classe et d'autres gens, l'emploi de l'expression « franglais». Cette expression m'a toujours dégoûtée, ce n'est pas parce qu'on appelle sa poubelle «la garbage» ou qu'on dit «que le show était awesome » qu'on ne parle pas français pour autant. (Le Réveil, $2005: 20$ ).

(41) Je fais jusse dire que les Francos, on est aussi raciste envers nous-mêmes qu'envers les anglophones. Je veux dire, moé je suis bilingue pis tous les Franco-Manitobains que je connais sont bilingues. Cossé tu veux ? L'anglais icitte, ça s'attrape comme un thume. Mais quand un ostie d'anglophone apprend le français, on se plie le cul en quatre pour le féliciter. Moé, parsonne me félicite pour avoir appris le français. Au contraire, je me fais chier dessus par les anglophones parce que je suis Franco pis je me fais chier dessus par les Francos parce que je parle mal. (Prescott, $2001: 50-51$ )

Ce type de protestation s'inscrit toutefois dans le cadre d'actes individuels et ponctuels, à visées souvent provocatrices, et ne se trouve pas relayé au niveau collectif. Au début des années 1990, ce discours revendicatif était loin de faire l'objet d'un consensus au sein de la communauté franco-manitobaine si on en croit les vives réactions qu'avaient suscitées les présentations de la pièce de Marc Prescott Sex, Lies et les Franco-Manitobains dont est tiré l'extrait (41). Certains membres de la communauté y avaient effectivement condamné l'emploi d'un langage jugé trop vulgaire et le recours à l'anglais ou au franglais y était pour beaucoup dans la réception négative de l'œuvre (cf. Entretien inédit avec Marc Prescott, juillet 2006). La pièce de Prescott, remontée à Saint-Boniface tout récemment (2009), n’a pas particulièrement provoqué de débats passionnels et on peut sans doute y voir une évolution des mentalités. Pourtant, rien n'indique qu'on assiste à l'heure actuelle à une prise en charge par les représentants de l'élite francophone d'un discours de légitimation de l'usage de variétés anglicisées de français. 


\section{Conclusion}

L'examen des principales attitudes linguistiques observées en contexte franco-manitobain nous a permis de constater que ces dernières correspondent à des schémas de perception courants au sein de l'espace francophone multilingue. Au Manitoba, comme dans d'autres communautés francocanadiennes aux prises avec un modèle normatif endogène ou exogène du français et en contact étroit avec la langue anglaise, les discours épilinguistiques portent sur la gamme d'usages qui se situent entre le pôle du «français correct» et celui de l'anglais. Ces discours révèlent diverses tendances qui, au-delà de celles traditionnellement mises au jour par les études sur les attitudes linguistiques, vont de la dévalorisation argumentée des variétés prestigieuses à la revendication de l'usage de variétés anglicisées du français chez certains acteurs de la communauté, cette dernière tendance demeurant essentiellement d'ordre individuel.

Comme chercheurs, nous souhaitons décrire et valoriser les phénomènes liés à la variation et au changement linguistiques. Ils s'associent en effet à la richesse patrimoniale et présentent un intérêt particulier pour l'étude du langage. Si le travail de description du linguiste peut contribuer à la connaissance des particularités des différentes variétés d'une même langue, il apparait toutefois que l'acceptation, voire la revendication de certaines variétés de la langue, comme les variétés anglicisées au Manitoba, reste du ressort de la communauté linguistique qui en fait usage. Ainsi, le processus de changement linguistique dû au contact des langues semble bien simple lorsqu'il est objectivement énoncé : «Il y a un temps - court - où les unités et les structures peuvent être considérées comme des emprunts ou comme des faits d'interlangue ou comme des innovations, il y a un temps où elles $\underline{\text { font partie }}^{9}$ du répertoire linguistique partagé par les membres du groupe » (Moreau, 1999 : 42). L'analyse des attitudes linguistiques en contexte francophone minoritaire montre pourtant que les normes subjectives jouent un rôle paralysant dans le processus d'intégration et de légitimation de ce type de changement linguistique. Au Manitoba, dans leur pratique quotidienne du français, les locuteurs francophones partagent bien des traits imputables au contact avec l'anglais et adoptent des comportements linguistiques en adéquation avec leur environnement bilingue. Comme nous l'avons montré, ces traits et ces comportements, à de rares exceptions près, sont cependant condamnés dans les discours. Les variétés anglicisées non légitimes qui apparaissent au bas de la hiérarchie linguistique constituent pourtant, pour certains francophones, leur seule ressource d'expression en français. Une trop grande dépréciation au sein même de la communauté linguistique de ces usages

\footnotetext{
${ }^{9}$ C'est Moreau qui souligne.
} 
linguistiques risque alors de « décourage[r] les locuteurs respectifs, les privant non seulement de leur conscience de soi, mais aussi de leur capacité d'expression en les faisant se taire » (Bochmann 2001 : 95) ... en français.

\section{Références bibliographiques}

Bochmann, Klaus. 2001. Notre langue, votre patois, leur baragouin : stéréotypes et représentations des langues. Hermès 30 : 93-102.

Boudreau, Annette. 1991. Les rapports que les jeunes Acadiens et Acadiennes entretiennent avec leur langue et avec la langue. Égalité $30: 13-37$.

http://www.umoncton.ca/egalite/article30.html

Boudreau, Annette et Françoise Gadet. 1998. Attitudes en situation minoritaire. L'exemple de l'Acadie. Dans Francophonies. Recueil d'études offert en hommage à Suzanne Lafage, sous la direction de Ambroise Queffélec. Nice : Didier Érudition. http://www.unice.fr/ILF-CNRS/ofcaf/12/Boudreau.htm

Entretien inédit avec Marc Prescott (juillet 2006).

Entrevue avec Jean-Denis Gendron. 2008. Qu'est-ce qu'il a, mon accent?, Québec Sciences, octobre $2008: 26-28$.

Forum de discussion sur le site Internet de Facebook: «Oui Yan, j’viens du CLR et je suis plus confortable en parlant en français ». Consulté le 22 décembre 2009. http:/ /www.facebook.com/group.php?gid=18686351606\&ref=search\&sid=1286753440.3154 $815349 . .1$

Fournier, Robert. 2001-2202. Français d'Icitte. Une grammaire basilectale. http://pages.infinit.net/noulawi/GFICh2JSP.htm

Gaborieau, Antoine. 2006. Le petit Gabi. Dictionnaire des anglicismes du Canada français. Saint-Boniface : Éditions des Plaines.

Hallion, Sandrine. 2000. Étude du français parlé au Manitoba. Thèse de doctorat, Université AixMarseille I - Université de Provence.

Lafontaine, Dominique. 1997. Attitudes linguistiques. Dans Sociolinguistique. Concepts de base, sous la direction de Marie-Louise Moreau, 56-60. Liège : Mardaga.

Laurendeau, Paul. 2004. Joual-franglais-français : la proximité dans l'épilinguistique. Dans Des langues collatérales - Problèmes linguistiques, sociolinguistiques et glottopolitiques de la proximité linguistique, sous la direction de Jean-Michel Éloy. La collection Espace discursif, T.II, 431-446. Paris : L'Harmattan.

Laurendeau, Paul. 2007. Avoir un méchant langage. Du comportement social dans les représentations épilinguistiques de la culture vernaculaire: le cas du Québec francophone. Glottopol 9 : 22-48.

http://www.univ-rouen.fr/dyalang/glottopol/telecharger/numero_9/gpl9_02laurendeau.pdf 
Le Réveil. 2005. Journal mensuel des étudiants du Collège universitaire de Saint-Boniface, vol. 44, édition 8 : $20-21$.

Léveillé, Roger. 2005. Rapport des écrivains franco-manitobains à la langue française. Dans Parade ou les autres, 83-110. Saint-Boniface : Les Éditions du Blé.

Moreau, Marie-Louise. 1999. Pluralité des normes et des appartenances: Convergences et divergences en situation pédagogique. Terminogramme 91-92 : 41-63.

Poirier, Claude, dir. 1998. Dictionnaire historique du français québécois. Monographies lexicographiques de québécismes. Sainte-Foy : Les Presses de l'Université Laval.

Prescott, Marc. 2001. Big, bullshit, sex, lies et les Franco-Manitobains. Dans la collection Rouge. SaintBoniface : Les Éditions du Blé.

Rodriguez, Liliane. 1984. Mots d'hier, mots d'aujourd'bui. Saint-Boniface : Éditions des Plaines. 